\title{
Associations Between Attention Deficit/ Hyperactivity Disorder and Internet Gaming Disorder Symptoms Mediated by Depressive Symptoms and Hopelessness Among College Students
}

\author{
Chen Chen ${ }^{1,2}$ \\ Si Dai ${ }^{2}$ \\ Lijuan $\mathrm{Shi}^{3}$ \\ Yidong Shen $\mathbb{D}^{2}$ \\ Jianjun $\mathrm{Ou} \mathbb{( D}^{2}$
}

\begin{abstract}
'Academy of Forensic Science, Shanghai Key Laboratory of Forensic Medicine, Key Laboratory of Forensic Science, Shanghai Forensic Service Platform, Key Laboratory of Forensic Science, Ministry of Justice, Shanghai, Shanghai, People's Republic of China; ${ }^{2}$ Department of Psychiatry, National Clinical Research Center for Mental Disorders, National Technology Institute on Mental Disorders, The Second Xiangya Hospital of Central South University, Changsha, Hunan, People's Republic of China; ${ }^{3}$ School of Education, Hunan University of Science and Technology, Xiangtan, Hunan, People's Republic of China
\end{abstract}

Purpose: The mediating role of depressive symptoms and hopelessness on the relationship between attention deficit/hyperactivity disorder (ADHD) and internet gaming disorder (IGD) symptoms has not yet been elucidated. This study assessed this mediating effect in college students.

Participants and Methods: Participants consisted of 1236 freshmen recruited at a local university. Symptoms of ADHD, IGD, depression and hopelessness were measured using self-report scales. The effect of ADHD on IGD mediated by depression and hopelessness was analyzed by Haye's PROCESS macro for SPSS (Model 6) using the bias-corrected percentile bootstrap method with 5000 bootstrapped samples.

Results: Symptoms of ADHD, depression and hopelessness were common in college students and were strongly associated with the appearance of IGD symptoms. ADHD symptoms of college students impacted their IGD symptoms directly and indirectly via depressive symptoms and hopelessness. The total effect of ADHD symptoms on IGD symptoms was 0.075 (standard error [SE]: 0.009 , 95\% confidence interval [CI] 0.057 , 0.093), with a direct effect of 0.037 (SE: $0.010,95 \%$ CI $0.018,0.056$, effect ratio: $49.3 \%$ ), and total indirect effect was 0.038 (SE: 0.005, 95\% CI 0.029, 0.050, effect ratio: 50.7\%).

Conclusion: Depressive symptoms and hopelessness can mediate the relationship between ADHD and IGD symptoms among college students. These findings point to the importance of evaluating ADHD, depressive and hopelessness symptoms for the prevention and treatment of adult IGD.

Keywords: attention deficit/hyperactivity disorder, internet gaming disorder, depression, hopelessness, mediation effect

\section{Introduction}

Department of Psychiatry, National Clinical Research Center for Mental Disorders, National Technology Institute on Mental Disorders, The Second Xiangya Hospital of Central South University,

No. 139 Middle Renmin Road, Furong

District, Changsha, 4I00II, Hunan,

People's Republic of China

Tel +86 I777586I486

Email oujianjun@csu.edu.cn
Attention deficit/hyperactivity disorder (ADHD) is a common neurodevelopmental disorder that is usually diagnosed in childhood. The prevalence of childhood ADHD is approximately $6 \%$, and patients are predominantly men. ${ }^{1}$ Symptoms of childhood ADHD can persist into adulthood in $40-50 \%$ of cases. ${ }^{2-4}$ Longitudinal evidence has suggested that the prevalence of adult ADHD ranges from 3\% to 5\% with gender balance, but some adults with ADHD lack a history of childhood 
ADHD. ${ }^{1,5-8}$ Adult ADHD is strongly associated with addictive behavior (for example, substance misuse and dependence), poor academic achievement and mental health, unemployment, and life impairment, ${ }^{1,9,10}$ thereby emphasizing the importance of investigating the underlying mechanisms.

Internet gaming disorder (IGD) is a common comorbidity of ADHD among young adults, characterized by the problematic use of internet games. ${ }^{11,12}$ Previous studies have shown that brain abnormalities in ADHD are associated with the biological susceptibility and addiction severity of IGD. ${ }^{13-15}$ Some neuropsychological characteristics, such as impulsivity and hostility, are also associated with the comorbidity of ADHD and IGD. ${ }^{16}$ For college students, the presence of probable ADHD is related to the severity of IGD symptoms and mediates the effect of immersive tendency on IGD.$^{11,17}$ Additionally, the association between ADHD and IGD may differ across cultures. ${ }^{18}$

Hopelessness refers to the cognitive patterns which characterized by the pessimistic attitude or the negative expectations about the future. ${ }^{19}$ Previous study indicated that adult with ADHD may expressed several common symptoms such as deficits in working memory, organizing, sustaining, and completing tasks. ${ }^{20}$ Consequently, failure to successfully complete tasks in daily life or work may bring frustration and hopelessness to adults with ADHD. In addition to this, many studies found that hopelessness is associated with addictive related behavior such as substance misuse. ${ }^{21,22}$ Similarly, the sense of hopelessness may lead individuals to become addicted to internet games to escape the challenges of reality. However, few studies have examined the role of hopelessness in the relationship between ADHD and IGD.

Depression is another common comorbidity of both ADHD and IGD. ${ }^{23,24}$ A longitudinal study has demonstrated that childhood ADHD increases the risk of depression later in life and is consistent with a causal effect of ADHD genetic liability on subsequent major depression. ${ }^{25}$ There has also been evidence of a positive correlation between depressive symptoms and ADHD symptoms in college students. ${ }^{26}$ Additionally, longitudinal studies have also pointed to a reciprocal relationship between the severity of IGD features and the level of depressive symptoms in children; ${ }^{27}$ in addition, depressive symptoms can positively predict IGD in adolescents, but not inversely. ${ }^{28}$ For college students, depression symptoms can predict the severity of IGD symptoms, mediate the relationship between childhood trauma and IGD, and augment its negative influence. ${ }^{11,29}$
This study was designed to assess the relationship between the symptoms of ADHD, IGD, and depression among college students. As cognitive biases can impact the relationship between depression and $\mathrm{ADHD}^{30,31}$ and are associated with the presence and psychotherapy effect of depression, ${ }^{32,33}$ hopelessness was also assessed in the study. The hypothesis tested was: ADHD symptoms are directly associated with IGD symptoms, and the effect can be indirectly enhanced through depressive and hopelessness symptoms.

\section{Participants and Methods Participants}

This cross-sectional study was conducted using simple cluster sampling method at a local university in Hunan province, China, between October 2018 and December 2018. Freshmen were recruited at the university according to the following criteria: (1) officially registered at the university and (2) enrolled full-time. All participants voluntarily signed a written informed consent prior to the investigation. The study was approved by the Clinical Research Ethics Committee of the Second Xiangya Hospital, Central South University, China. This study was conducted in accordance with the Declaration of Helsinki.

A total of 1309 questionnaires were distributed, and 1236 participants completed and returned valid questionnaires, resulting in a response rate of $94.4 \%$. The mean age of college students was 20.39 (0.99)-years-old, and their paternal and maternal mean age was 46.60 (4.07) and 44.53 (4.01), respectively. The proportion of females was $60.2 \%$, while that of males was $39.8 \%$. Most participants were of Han ethnicity (91.9\%), living in middle to high income families $(96.3 \%)$, and had no family history of mental disorders $(92.7 \%)$.

\section{Measurements}

Sociodemographic characteristics of the college students were assessed using a self-designed structured questionnaire, including the age, sex, ethnicity, parents' age and education, family economic level, and family history of mental illness.

The Adult ADHD Self-Report Screening Scale for DSM-5 (ASRS-5) was used to screen for ADHD symptoms in this study. ${ }^{34}$ It consists of a DSM-5 criterion updated scale of the widely used World Health Organization Adult ADHD Self-Report Scale, characterized by a few number of items (six items), ease of scoring, and excellent operating value [sensitivity (91.4-91.9\%), 
specificity (96.0-74.0\%), area under the curve (0.94-0.83), and positive predictive value (67.3-82.8\%)]. Each item of the scale assesses the frequency of ADHD symptoms as the patients experience them over the past 6 months, with answers ranging from never to very often ( 5 levels). The total score ranges from 0 to 24 and is calculated by adding the response scores for all items. Patients with a score $\geq 14$ were considered to have probable ADHD. ${ }^{34}$ Proprietary scoring rules with the permission of New York University were applied in this study.

The dichotomous score of the Internet Gaming Disorder Scale, 9-item (IGDS-9) was used to assess IGD based on the DSM-5 criteria. $^{35}$ It consists of a self-report scale that assesses IGD symptoms over the past 12 months. Each item on the scale is rated with either no (0) or yes (1). The summed scores of the IGDS-9 range from 0 to 9 , and can best be used to assess the prevalence of IGD when the DSM-5 cutoff point of five or more is used. The scale provides a valid and reliable measure of IGD with good diagnostic accuracy that can be used for research and diagnostic purposes among male and female gamers of all ages. ${ }^{35}$

The Quick Inventory of Depressive SymptomatologySelf-Report (QIDS-SR) was used to assess college students' symptoms of depression. ${ }^{36}$ It consists of a self-report scale and contains nine types of depressive symptoms that are consistent with the DSM-4 criterion domains. The scale consists of 16 items rated on a 4-point Likert scale. Each item assesses the severity of depressive symptoms that occurred over the past 7 days. The total score ranges from 0 to 27 , with higher scores indicating severe symptoms of depression. Patients with a score $\geq 6$ were considered to have mild to very severe depression. The Chinese version of the QIDS-SR has been validated with good psychometric estimates (Cronbach's $\alpha=0.76-0.82) .{ }^{37}$

The Beck Hopelessness Scale (BHS) was used to assess hopelessness in this study. ${ }^{38}$ It consists of a 20 -item selfreport scale with a true-false response format. It contains 11 negatively worded items (yes $=1$ and no $=0$ ) and nine positively worded items (yes $=0$ and no $=1$ ). The sum scores of BHS range from 0 to 20 , with a higher score indicating a higher level of hopelessness (normal range: 0-3, mild hopelessness: 4-8, moderate hopelessness: 9-14, severe hopelessness: >14). This scale has been widely used by college students and has good reliability and validity. ${ }^{39,40}$ It has also been used to measure hopelessness among the Chinese population. ${ }^{41,42}$

\section{Statistical Analysis}

A correlation analysis was performed using Spearman correlation when the continuous variables were abnormally distributed. To test this hypothesized mediation, data were analyzed by Haye's PROCESS macro for SPSS (Model 6) using the bias-corrected percentile bootstrap method with 5000 bootstrapped samples. Three equations were used to assess the potential mediating role of hopelessness and depressive symptoms in the relationship between ADHD and IGD after controlling for the effect of age and sex through multiple linear regression. Data analyses were performed using SPSS (version 25.0; Armonk, NY, USA) with a significance level of $p<0.05$.

\section{Results}

\section{Socio-Demographic and Clinical Characteristics}

The prevalence of being at high risk of ADHD and IGD symptoms was $12 \%$ and $4.9 \%$, respectively. The percentage of participants with moderate to severe depression was $6 \%$, and none of them had very severe depression. Approximately $12.7 \%$ of the participants reported moderate to severe hopelessness. Table 1 represents the detailed characteristics of the participants.

\section{Correlations Between IGD and Other Symptoms}

None of the continuous variables satisfied a normal distribution. Positive correlations were found between IGD and ADHD symptoms $(r=0.227, p<0.001)$, hopelessness $(\mathrm{r}=0.226, \mathrm{p}<0.001)$, and depressive symptoms $(\mathrm{r}=0.265$, $\mathrm{p}<0.001)$. ADHD symptoms were also significantly correlated with depressive symptoms $(r=0.369, \mathrm{p}<0.001)$ and hopelessness $(r=0.279, p<0.001)$. Additionally, depressive symptoms and hopelessness were also highly correlated $(\mathrm{r}=0.414, \mathrm{p}<0.001)$ (Table 2).

\section{Mediating Role of Depressive Symptoms and Hopelessness}

A mediation model with depressive symptoms and hopelessness as mediators between ADHD and IGD symptoms was tested (Figure 1). The total effect of ADHD symptoms on IGD symptoms was 0.075 (standard error [SE]: 0.009, 95\% confidence interval [CI] 0.057, 0.093), with a direct effect of 0.037 (SE: $0.010,95 \%$ CI 0.018, 0.056, effect ratio: 49.3\%); the total indirect effect was 0.038 (SE: $0.005,95 \%$ CI 0.029 , 
Table I Socio-Demographics and Clinical Characteristics of the Sample

\begin{tabular}{|c|c|c|}
\hline & $\mathbf{N}$ & $\%$ \\
\hline \multicolumn{3}{|l|}{ Gender } \\
\hline Male & 492 & 39.8 \\
\hline Female & 744 & 60.2 \\
\hline \multicolumn{3}{|l|}{ Ethnic } \\
\hline Han & 1136 & 91.9 \\
\hline Others & 100 & 8.1 \\
\hline \multicolumn{3}{|l|}{ Perceived Family Economic Level } \\
\hline Low & 46 & 3.7 \\
\hline Middle & 581 & 47.0 \\
\hline High & 609 & 49.3 \\
\hline \multicolumn{3}{|l|}{ Family History of Mental Disorder } \\
\hline Yes & 90 & 7.3 \\
\hline No & 1146 & 92.7 \\
\hline \multicolumn{3}{|l|}{ Paternal Education } \\
\hline Under the high school & 687 & 55.6 \\
\hline High school & 308 & 24.9 \\
\hline University and above & 241 & 19.5 \\
\hline \multicolumn{3}{|l|}{ Maternal Education } \\
\hline Under the high school & 807 & 65.3 \\
\hline High school & 232 & 18.8 \\
\hline University and above & 197 & 15.9 \\
\hline \multicolumn{3}{|l|}{ ASRS-5 with Positive Score } \\
\hline Yes & 148 & 12.0 \\
\hline No & 1088 & 88.0 \\
\hline \multicolumn{3}{|l|}{ IGDS-9 with Positive Score } \\
\hline Yes & 61 & 4.9 \\
\hline No & 1175 & 95.1 \\
\hline \multicolumn{3}{|l|}{ QIDS-SR } \\
\hline No depression & 809 & 65.5 \\
\hline Mild depression & 353 & 28.6 \\
\hline Moderate depression & 72 & 5.8 \\
\hline Severe depression & 2 & 0.2 \\
\hline Very severe depression & 0 & 0 \\
\hline \multicolumn{3}{|l|}{$\mathrm{BHS}$} \\
\hline Normal range & 450 & 36.4 \\
\hline Mild hopelessness & 629 & 50.9 \\
\hline Moderate hopelessness & 154 & 12.5 \\
\hline Severe hopelessness & 3 & 0.2 \\
\hline
\end{tabular}

0.050, effect ratio: $50.7 \%$ ). With regard to indirect effects, values of 0.016 (SE: $0.004,95 \%$ CI $0.009,0.023$, effect ratio: $21.3 \%$ ) was found in the pathway of ADHD symptoms hopelessness - IGD symptoms, 0.017 (SE: 0.004, 95\% CI $0.010,0.024$, effect ratio: $22.7 \%$ ) in the pathway of ADHD symptoms - depressive symptoms - IGD symptoms, and
Table 2 Correlations Between ADHD, Hopelessness, Depressive and IGD Symptoms

\begin{tabular}{|l|l|c|c|c|}
\hline & & $\mathbf{I}$ & $\mathbf{2}$ & $\mathbf{3}$ \\
\hline $\mathrm{I}$ & IGDS-9 total score & 1.000 & & \\
2 & ASRS-5 total score & $0.227^{* * *}$ & 1.000 & \\
3 & BHS total score & $0.226^{* * *}$ & $0.279 * * *$ & 1.000 \\
4 & QIDS-SR total score & $0.265^{* * *}$ & $0.369^{* * *}$ & $0.414^{* * *}$ \\
\hline
\end{tabular}

Note: $* * * p<0.001$.

0.006 (SE: $0.002,95 \%$ CI 0.004, 0.009, effect ratio: $8.0 \%$ ) in the pathway of ADHD symptoms - hopelessness - depressive symptoms - IGD symptoms (Table 3 ). The direct effect accounted for $49.3 \%$ of the total effect, this ratio seems to be accepted. Therefore, the results of this study are reliable and persuasive.

\section{Discussion}

This study assessed the mediating role of depressive symptoms and hopelessness on the relationship between ADHD and IGD symptoms. Results demonstrated that college students' ADHD symptoms impacted their IGD symptoms directly and indirectly via depressive symptoms and hopelessness. The ratios of the direct and indirect effects are roughly equal. These findings are meaningful for understanding the development of IGD and are useful for preventing and treating IGD.

ADHD is directly associated with addictive behavior based on common biological mechanisms. In this study, ADHD symptoms were significantly associated with IGD symptoms, which is consistent with the findings of previous studies conducted across different populations. ${ }^{11,43,44}$ The comorbidity of ADHD and IGD points to a common biological mechanism. Previous magnetic resonance spectroscopy and imaging studies have found that ADHD and IGD patients were both characterized by decreased $\mathrm{N}$-acetyl-aspartate levels within the frontal lobe, altered functional connectivity of the posterior cingulate cortex, and gray matter volume. ${ }^{13,14,45}$ A recent study also found that an aberrant increase in circuits related to inhibitory function or sensory integration can indicate an association between comorbid ADHD and addiction severity in IGD. ${ }^{15}$ Additionally, impulsiveness, a neuropsychological trait, has also been found to be associated with ADHD and IGD. ${ }^{13,16}$

Several previous studies found that high level of hopelessness was associated with addictive related behavior, such as alcohol use, tobacco addiction, and drinking. ${ }^{46,47}$ Besides this, higher levels of hopelessness were found 


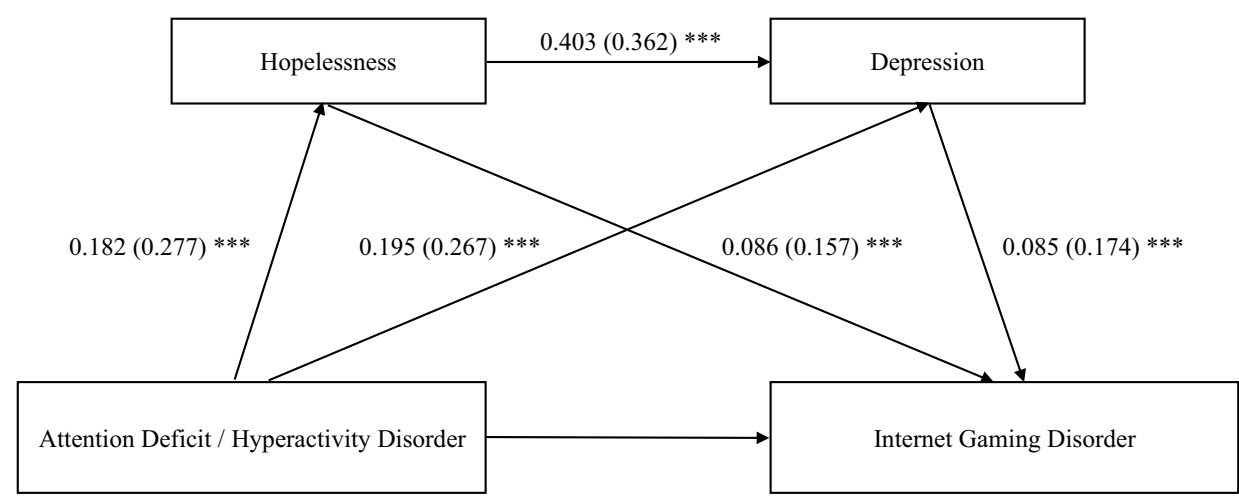

Direct effect: $0.037(0.103) * * *$

Total effect: $0.075(0.210) * * *$

Figure I The effect of ADHD symptoms on IGD symptoms through hopelessness and depressive symptoms.

Notes: Unstandardized coefficient-beta (standardized coefficient- $\beta$ ), ${ }^{* * *} \mathrm{p}<0.00 \mathrm{I}$.

among heroin addicts and problem internet users than in the control group. ${ }^{48,49}$ In the current study, we also found the significant association between hopelessness and IGD symptoms, add the evidence linking hopelessness to addictive behaviors. In addition to this, hopelessness was found could mediate the effect of ADHD on IGD symptoms, which accounted for $21.3 \%$ of the total effect. It is possible that in adult with ADHD symptoms, the feelings of hopelessness and the negative expectation about future might impaired the ability to effectively complete tasks or solve problems, which in turn, may lead to addicted to internet games among these people. ${ }^{50}$

The indirect effects of emotion and negative cognition also play an important role in the association between ADHD and IGD symptoms. According to our results, indirect effects accounted for half of the total effects on the association between ADHD and IGD symptoms, while the indirect effects through depressive symptoms or hopelessness were relatively equal. These findings highlight the importance of psychosocial factors as mediators between ADHD and IGD, and reveals the equal values of emotion and negative cognition. Although the underlying mechanisms remain unclear, previous studies have provided some evidence to support our speculation. Individuals with ADHD often experience interpersonal tension, setbacks, and stress due to poor function, thereby leading to susceptibility to depressive symptoms and hopelessness. ${ }^{9,10}$ Addictive behaviors, such as online gaming addiction, are sometimes considered compensatory or self-medicating behaviors for ADHD, depression, and other mental disorders. ${ }^{51-53}$

Results of this study have significant clinical implications. Our findings suggest that symptoms of ADHD, depression, and hopelessness are common in college students and are strongly associated with the appearance of IGD symptoms. This provides a target for the prevention of IGDs. The evaluation and treatment of ADHD, depressive and hopelessness symptoms in IGD patients may be important since these conditions impact initial and sustained responses to treatment for IGD. ${ }^{54,55}$ Pharmacotherapy and psychotherapy have been found to have significant effects on adult ADHD and depressive symptoms, while the latter has a better effect on symptoms of hopelessness. ${ }^{33,56,57}$ Some pharmacotherapeutic and

Table 3 Mediation Results for ADHD, Hopelessness, Depressive and IGD Symptoms

\begin{tabular}{|l|c|c|c|c|c|c|}
\hline & Path & \multirow{2}{*}{ Effect } & \multirow{2}{*}{ SE } & \multicolumn{2}{|c|}{ 95\% CI } & Effect Ratio \\
\cline { 3 - 6 } & & & & Low & UP \\
\hline Total effect & & 0.075 & 0.009 & 0.057 & 0.093 & $100.0 \%$ \\
Direct effect & & 0.037 & 0.010 & 0.018 & 0.056 & $49.3 \%$ \\
Total indirect effect & & 0.038 & 0.005 & 0.029 & 0.050 & $50.7 \%$ \\
Indirect effect I & ASRS $\rightarrow$ BHS $\rightarrow$ IGD & 0.016 & 0.004 & 0.009 & 0.023 & $21.3 \%$ \\
Indirect effect 2 & ASRS $\rightarrow$ QIDS $\rightarrow$ IGD & 0.017 & 0.004 & 0.010 & 0.024 & $22.7 \%$ \\
Indirect effect 3 & ASRS $\rightarrow$ BHS $\rightarrow$ QIDS $\rightarrow$ IGD & 0.006 & 0.002 & 0.004 & 0.009 & $8.0 \%$ \\
\hline
\end{tabular}


psychotherapeutic strategies have been developed to treat substance use in adults with ADHD. ${ }^{58,59}$ It may be feasible to develop a comprehensive treatment for adults with IGD combined with ADHD based on these existing treatments.

This study also had some limitations. First, only selfreported scales were used to measure mental symptoms. However, the ability of participants to accurately estimate their mental symptoms remains questionable. Second, as it was a cross-sectional study, the direction of causality among the variables could not be accurately determined, and instead could only theoretically hypothesized. Third, we only investigated college freshmen; whether these findings are consistent across grade levels remains uncertain. Fourth, because the measured participants are ordinary college freshmen, it is not clear that whether the research results are also applicable to clinical patients and whether they can provide guidance for psychiatrists. Fifth, ASRS-5 and IGDS-9 used in this study is simply relying on 6 items and cut-off score which selected or set in the US, it has not undergone strict localization validation research and reliability and validity analysis. However, these two scales used in this study were screened on the basis of DSM-5 diagnosis. In China, DSM-5 innovates in the coding, classification and diagnosis of mental disorders, has a far-reaching impact on many professional disciplines, and has been recognized and praised by scholars. Therefore, although the scale used in this study did not verify the scale's local research in China, its results still have a certain reliability. Future work should determine whether the patients have IGD, ADHD, major depression, and other psychiatric disorders through rigorous clinical diagnosis, better identify direct causal inferences by utilizing longitudinal designs, and explore whether the associations reported here change over time.

\section{Conclusion}

Depressive symptoms and hopelessness can mediate the relationship between ADHD and IGD symptoms among college students. The findings point to the importance of evaluating ADHD, depressive and hopelessness symptoms in college student with IGD. These conditions may be targets for prevention and treatment of adult IGD.

\section{Funding}

The study was supported by the National Natural Science Foundation of China (No.81974217 and 81901388), the Natural Science Foundation of Hunan Province (nos. 2020JJ5825 and 2020JJ5830), the Humanity and Social Science Youth Foundation of the Ministry of Education
(17YJC190022), the Scientific Research Fund of Hunan Provincial Education Department (18B219).

\section{Disclosure}

The authors declared no potential conflicts of interest with respect to the research, authorship, and/or publication of this article.

\section{References}

1. Moffitt TE, Houts R, Asherson P, et al. Is adult ADHD a childhood-onset neurodevelopmental disorder? Evidence from a four-decade longitudinal cohort study. $\mathrm{Am} J$ Psychiatry. 2015;172:967-977. doi:10.1176/appi.ajp.2015.14101266

2. Sibley MH, Mitchell JT, Becker SP. Method of adult diagnosis influences estimated persistence of childhood ADHD: a systematic review of longitudinal studies. Lancet Psychiatry. 2016;3:1157-1165. doi:10.1016/S2215-0366(16)30190-0

3. Biederman J, Mick E, Faraone SV. Age-dependent decline of symptoms of attention deficit hyperactivity disorder: impact of remission definition and symptom type. Am J Psychiatry. 2000;157:816-818.

4. Kessler RC, Adler LA, Barkley R, et al. Patterns and predictors of attention-deficit/hyperactivity disorder persistence into adulthood: results from the national comorbidity survey replication. Biol Psychiatry. 2005;57:1442-1451.

5. De Zwaan M, Gruss B, Muller A, et al. The estimated prevalence and correlates of adult ADHD in a German community sample. Eur Arch Psychiatry Clin Neurosci. 2012;262:79-86.

6. Fayyad J, De Graaf R, Kessler R, et al. Cross-national prevalence and correlates of adult attention-deficit hyperactivity disorder. $\mathrm{Br}$ J Psychiatry. 2007;190:402-409.

7. Kessler RC, Adler L, Barkley R, et al. The prevalence and correlates of adult ADHD in the United States: results from the national comorbidity survey replication. Am J Psychiatry. 2006;163:716-723.

8. Vnukova M, Ptacek R, Dechterenko F, et al. Prevalence of ADHD symptomatology in adult population in the czech republic-A national study. J Atten Disord. 2020. doi:10.1177/1087054720934042

9. Agnew-Blais JC, Polanczyk GV, Danese A, et al. Young adult mental health and functional outcomes among individuals with remitted, persistent and late-onset ADHD. Br J Psychiatry. 2018;213:526-534.

10. Erskine HE, Norman RE, Ferrari AJ, et al. Long-term outcomes of attention-deficit/hyperactivity disorder and conduct disorder: a systematic review and meta-analysis. J Am Acad Child Adolesc Psychiatry. 2016;55:841-850. doi:10.1016/j.jaac.2016.06.016

11. Evren C, Evren B, Dalbudak E, et al. Relationships of Internet addiction and Internet gaming disorder symptom severities with probable attention deficit/hyperactivity disorder, aggression and negative affect among university students. Atten Defic Hyperact Disord. 2019;11:413-421. doi:10.1007/s12402-019-00305-8

12. Dullur P, Krishnan V, Diaz AM. A systematic review on the intersection of attention-deficit hyperactivity disorder and gaming disorder. $J$ Psychiatr Res. 2021;133:212-222. doi:10.1016/j. jpsychires.2020.12.026

13. Lee D, Lee J, Lee JE, et al. Altered functional connectivity in default mode network in Internet gaming disorder: influence of childhood ADHD. Prog Neuropsychopharmacol Biol Psychiatry. 2017;75:135-141. doi:10.1016/j.pnpbp.2017.02.005

14. Lee D, Namkoong K, Lee J, et al. Preliminary evidence of altered gray matter volume in subjects with internet gaming disorder: associations with history of childhood attention-deficit/hyperactivity disorder symptoms. Brain Imaging Behav. 2019;13:660-668. doi:10.1007/s11682-018-9872-6 
15. Kim M, Kim D, Bae S, et al. Aberrant structural network of comorbid attention deficit/hyperactivity disorder is associated with addiction severity in internet gaming disorder. Neuroimage Clin. 2020;27:102263. doi:10.1016/j.nicl.2020.102263

16. Yen JY, Liu TL, Wang PW, et al. Association between Internet gaming disorder and adult attention deficit and hyperactivity disorder and their correlates: impulsivity and hostility. Addict Behav. 2017;64:308-313. doi:10.1016/j.addbeh.2016.04.024

17. Jung D, Shim EJ, Park H, et al. The association between excessive internet gaming behavior and immersive tendency, mediated by attention deficit/hyperactivity disorder symptoms, in korean male university students. Psychiatry Investig. 2020;17:403-411. doi:10.30773/ pi. 2019.0173

18. Stavropoulos V, Adams BLM, Beard CL, et al. Associations between attention deficit hyperactivity and internet gaming disorder symptoms: is there consistency across types of symptoms gender and countries?. Addict Behav Rep. 2019;9:100158.

19. Kazdin AE, Rodgers A, Colbus D. The hopelessness scale for children: psychometric characteristics and concurrent validity. $J$ Consult Clin Psychol. 1986;54:241-245. doi:10.1037/0022-006X.54.2.241

20. Kessler RC, Green JG, Adler LA, et al. Structure and diagnosis of adult attention-deficit/hyperactivity disorder: analysis of expanded symptom criteria from the Adult ADHD clinical diagnostic scale. Arch Gen Psychiatry. 2010;67:1168-1178. doi:10.1001/archgenpsy chiatry.2010.146

21. Krank M, Stewart SH, O’Connor R, et al. Structural, concurrent, and predictive validity of the substance use risk profile scale in early adolescence. Addict Behav. 2011;36:37-46. doi:10.1016/j. addbeh.2010.08.010

22. Mackinnon SP, Kehayes IL, Clark R, et al. Testing the four-factor model of personality vulnerability to alcohol misuse: a three-wave, one-year longitudinal study. Psychol Addictive Behav. 2014;28:1000-1012. doi:10.1037/a0037244

23. Ostinelli EG, Zangani C, Giordano B, et al. Depressive symptoms and depression in individuals with internet gaming disorder: a systematic review and meta-analysis. $J$ Affect Disord. 2021;284:136-142. doi:10.1016/j.jad.2021.02.014

24. Daviss WB. A review of co-morbid depression in pediatric ADHD: etiology, phenomenology, and treatment. $J$ Child Adolesc Psychopharmacol. 2008;18:565-571. doi:10.1089/cap.2008.032

25. Riglin L, Leppert B, Dardani C. et al. ADHD and depression: investigating a causal explanation. Psychol Med;2020. 1-8. doi:10.1017/ S0033291720000665

26. Panevska LS, Zafirova-Ivanovska B, Vasilevska K, et al. Relationship between ADHD and depression among university students in macedonia. Mater Sociomed. 2015;27:18-21. doi:10.5455/ msm.2014.27.18-21

27. Jeong H, Yim HW, Lee SY, et al. Reciprocal relationship between depression and Internet gaming disorder in children: a 12-month follow-up of the iCURE study using cross-lagged path analysis. $J$ Behav Addict. 2019;8:725-732. doi:10.1556/2006.8.2019.74

28. Teng Z, Pontes HM, Nie Q, et al. Depression and anxiety symptoms associated with internet gaming disorder before and during the COVID-19 pandemic: a longitudinal study. $J$ Behav Addict. 2021;10:169-180. doi:10.1556/2006.2021.00016

29. Shi L, Wang Y, Yu H, et al. The relationship between childhood trauma and Internet gaming disorder among college students: a structural equation model. $J$ Behav Addict. 2020;9:175-180. doi: $10.1556 / 2006.2020 .00002$

30. Shapero BG, Gibb BE, Archibald A, et al. Risk factors for depression in adolescents with ADHD: the impact of cognitive biases and stress. $J$ Atten Disord. 2021;25:340-354.

31. Strohmeier CW, Rosenfield B, DiTomasso RA, et al. Assessment of the relationship between self-reported cognitive distortions and adult ADHD, anxiety, depression, and hopelessness. Psychiatry Res. 2016;238:153-158.
32. Alloy LB, Abramson LY, Metalsky GI, et al. The hopelessness theory of depression: attributional aspects. Br J Clin Psychol. 1988;27:5-21.

33. Cuijpers P, de Beurs DP, van Spijker BA, et al. The effects of psychotherapy for adult depression on suicidality and hopelessness: a systematic review and meta-analysis. $J$ Affect Disord. 2013;144:183-190.

34. Ustun B, Adler LA, Rudin C, et al. The world health organization adult attention-deficit/hyperactivity disorder self-report screening scale for DSM-5. JAMA Psychiatry. 2017;74:520-526.

35. Lemmens JS, Valkenburg PM, Gentile DA. The internet gaming disorder scale. Psychol Assess. 2015;27:567-582.

36. Rush AJ, Trivedi MH, Ibrahim HM, et al. The 16-Item quick inventory of depressive symptomatology (QIDS), clinician rating (QIDS-C), and self-report (QIDS-SR): a psychometric evaluation in patients with chronic major depression. Biol Psychiatry. 2003;54:573-583.

37. Liu J, Xiang Y-T, Wang G, et al. Psychometric properties of the chinese versions of the quick inventory of depressive symptomatology - clinician rating (C-QIDS-C) and self-report (C-QIDS-SR). $J$ Affect Disord. 2013;147:421-424.

38. Beck AT, Weissman A, Lester D, et al. The measurement of pessimism: the hopelessness scale. $J$ Consult Clin Psychol. 1974;42:861-865.

39. Boduszek D, Dhingra K. Construct validity of the beck hopelessness scale (BHS) among university students: a multitrait-multimethod approach. Psychol Assess. 2016;28:1325-1330.

40. Troister T, D’Agata MT, Holden RR. Suicide risk screening: comparing the beck depression inventory-II, beck hopelessness scale, and psychache scale in undergraduates. Psychol Assess. 2015;27:1500-1506.

41. Zhang J, Li Z. The association between depression and suicide when hopelessness is controlled for. Compr Psychiatry. 2013;54:790-796.

42. Tang L, Li Z, Pang Y. The differences and the relationship between demoralization and depression in Chinese cancer patients. Psychooncology. 2020;29:532-538.

43. Vally Z. Symptoms of internet gaming disorder, inattention, and impulsivity: a cross-sectional study conducted in the United Arab Emirates. Psychiatr Q. 2021;92:301-310.

44. Jeong H, Yim HW, Lee SY, et al. Factors associated with severity, incidence or persistence of internet gaming disorder in children and adolescents: a 2-year longitudinal study. Addiction. 2020;116 (7):1828-1838.

45. Bae S, Han DH, Kim SM, et al. Neurochemical correlates of internet game play in adolescents with attention deficit hyperactivity disorder: a proton magnetic resonance spectroscopy (MRS) study. Psychiatry Res Neuroimaging. 2016;254:10-17.

46. Ilhan IO, Demirbas H, Dogan YB. Psychosocial factors in alcohol use-related problems of working youth. Subst Use Misuse. 2007;42:1537-1544.

47. Bolland JM. Hopelessness and risk behaviour among adolescents living in high-poverty inner-city neighbourhoods. $J$ Adolesc. 2003;26:145-158.

48. Iliceto P, Pompili M, Girardi P, et al. Hopelessness, temperament, and health perception in heroin addicts. J Addict Dis. 2010;29:352-358.

49. Alpaslan AH, Soylu N, Kocak U, et al. Problematic Internet use was more common in Turkish adolescents with major depressive disorders than controls. Acta Paediatrica (Oslo, Norway: 1992). 2016;105:695-700.

50. Castellanos-Ryan N, Conrod P. Personality and substance misuse: evidence for a four-factor model of vulnerability. In drug abuse and addiction in medical illness: causes, consequences and treatment (Vol. 9781461433750). Springer New York. 2012;47-62.

51. Liebrenz M, Frei A, Fisher CE, et al. Adult attention-deficit/hyperactivity disorder and nicotine use: a qualitative study of patient perceptions. BMC Psychiatry. 2014;14:141. 
52. Bruijnzeel AW, Repetto M, Gold MS. Neurobiological mechanisms in addictive and psychiatric disorders. Psychiatr Clin North Am. 2004;27:661-674.

53. Chang YH, Chang KC, Hou WL, et al. Internet gaming as a coping method among schizophrenic patients facing psychological distress. $J$ Behav Addict. 2020;9:1022-1034.

54. Lee J, Bae S, Kim BN, et al. Impact of attention-deficit/hyperactivity disorder comorbidity on longitudinal course in Internet gaming disorder: a 3-year clinical cohort study. J Child Psychol Psychiatry. 2021. doi: $10.1111 /$ jcpp. 13380

55. Han DH, Yoo M, Renshaw PF, et al. A cohort study of patients seeking Internet gaming disorder treatment. $J$ Behav Addict. 2018;7:930-938.
56. Perugi G, Pallucchini A, Rizzato S, et al. Current and emerging pharmacotherapy for the treatment of adult attention deficit hyperactivity disorder (ADHD). Expert Opin Pharmacother. 2019;20:1457-1470.

57. Malhi GS, Mann JJ. Depression. Lancet. 2018;392:2299-2312.

58. Young S, Woodhouse E. Assessment and treatment of substance use in adults with ADHD: a psychological approach. J Neural Transm (Vienna). 2021;128(7):1099-1108.

59. Perugi G, Pallucchini A, Rizzato S, et al. Pharmacotherapeutic strategies for the treatment of attention-deficit hyperactivity (ADHD) disorder with comorbid substance-use disorder (SUD). Expert Opin Pharmacother. 2019;20:343-355.

\section{Publish your work in this journal}

Neuropsychiatric Disease and Treatment is an international, peerreviewed journal of clinical therapeutics and pharmacology focusing on concise rapid reporting of clinical or pre-clinical studies on a range of neuropsychiatric and neurological disorders. This journal is indexed on PubMed Central, the 'PsycINFO' database and CAS, and is the official journal of The International Neuropsychiatric Association (INA). The manuscript management system is completely online and includes a very quick and fair peer-review system, which is all easy to use. Visit http://www.dovepress.com/testimonials.php to read real quotes from published authors. 